\title{
DIFFEOMORPHISMS AND VOLUME-PRESERVING EMBEDDINGS OF NONCOMPACT MANIFOLDS
}

\author{
BY \\ R. E. GREENE ${ }^{1}$ AND K. SHIOHAMA ${ }^{2}$
}

\begin{abstract}
ABSTRACr. The theorem of J. Moser that any two volume elements of equal total volume on a compact manifold are diffeomorphism-equivalent is extended to noncompact manifolds: A necessary and sufficient condition (equal total and same end behavior) is given for diffeomorphism equivalence of two volume forms on a noncompact manifold. Results on the existence of embeddings and immersions with the property of inducing a given volume form are also given. Generalizations to nonorientable manifolds and manifolds with boundary are discussed.
\end{abstract}

The topics of this paper are the action of the diffeomorphism group of a noncompact paracompact oriented manifold on the space of $C^{\infty}$ volume forms on the manifold and the existence of volume-form-preserving embeddings of such manifolds into euclidean spaces. The results are essentially generalizations to the case of noncompact manifolds of a theorem of Moser [6] and a corollary of that theorem. The theorem is that if $M$ is a compact connected oriented manifold and if $\omega$ and $\tau$ are $C^{\infty}$ volume forms on $M$ such that $\int_{M} \omega=\int_{M} \tau$ then there is a $C^{\infty}$ diffeomorphism $\varphi: M \rightarrow M$ such that $\varphi^{*} \tau=\omega$. The corollary is that if $\omega$ is a $C^{\infty}$ volume form on such a manifold and if there is a $C^{\infty}$ embedding (immersion) of $M$ into a particular euclidean space $R^{k}$ then there is a $C^{\infty}$ embedding (immersion) of $M$ into that euclidean space with the property that the volume form of the Riemannian metric induced on $M$ by the embedding (immersion) is $\omega$. Such an embedding or immersion will be called volume-preserving hereafter. The corollary as stated follows from the theorem as follows: If $E: M \rightarrow R^{k}$ is an embedding (immersion) and $\omega_{E}=$ the volume form of the Riemannian metric induced by $E$ then for some positive real number $\lambda, \int_{M} \omega_{\lambda E}=\int_{M} \omega$. If $\varphi: M \rightarrow M$ is a diffeomorphism such that $\varphi^{*} \omega_{\lambda E}=\omega$, the existence of which is guaranteed by

Received by the editors September 7, 1977 and, in revised form, April 3, 1978 and November 13, 1978.

AMS (MOS) subject classifications (1970). Primary 57D50, 57D40; Secondary 53C40, 58A10.

Key words and phrases. Volume form, diffeomorphism, ends of a manifold, volume-preserving embedding, isometric embedding, odd forms.

${ }^{1}$ Research supported by the National Science Foundation under grant MCS77-03976 and the Institute for Advanced Study.

${ }^{2}$ Research supported by the Royal Danish Science Foundation. 
Moser's theorem, then $\lambda E \circ \varphi: M \rightarrow R^{k}$ is the required embedding (immersion).

The result obtained for noncompact manifolds, analogous to the first result, is:

THEOREM 1. If $M$ is a noncompact connected oriented manifold and if $\omega$ and $\tau$ are volume forms on $M$ with $\int_{M} \omega=\int_{M} \tau<+\infty$ and if each end of the manifold $M$ has finite $\omega$ volume if it has finite $\tau$ volume and infinite $\omega$ volume if it has infinite $\tau$ volume, then there is a diffeomorphism $\varphi: M \rightarrow M$ such that $\varphi^{*} \omega=\tau$.

The definition of finiteness and infiniteness of volume of an end will be given later as well as an example showing that the end condition cannot be omitted.

The generalization of the embedding-immersion result to the case of noncompact manifolds is the following result:

THEOREM 2. If a noncompact connected oriented manifold $M$ of dimension greater than 1 has a proper embedding (immersion) in a euclidean space $R^{k}$ of dimension greater than the dimension of $M$, then for any volume form $\omega$ on $M$ there is a proper embedding (immersion) of $M$ in $R^{k}$ which is volume-preserving for $\omega$.

Results corresponding to Theorems 1 and 2 hold for noncompact manifolds with boundary (see [1] and [2] for the case of compact manifolds with boundary). These results are discussed later in the paper, but for the sake of brevity they are omitted in this introduction.

In [4], it is shown that if $\omega$ and $\tau$ are real analytic volume forms on a real analytic noncompact oriented manifold $M$, then there is a real analytic diffeomorphism $\varphi: M \rightarrow M$ with $\varphi^{*} \omega=\tau$ if (and only if) there is a $C^{\infty}$ diffeomorphism $\psi: M \rightarrow M$ such that $\psi^{*} \omega=\tau$. Thus the real analytic problem reduces to the $C^{\infty}$ problem discussed in the present paper.

The hypothesis that $M$ have a proper immersion in $\mathbf{R}^{k}(k>$ dimension $M)$ in Theorem 2 can be replaced by the hypothesis that $M$ have an immersion in $\mathbf{R}^{k}$, not necessarily proper: the results of Hirsch [5] show that if any immersion exists then a proper immersion exists (here $k \rightarrow$ dimension $M$ is needed). Specifically, a $C^{\infty}$ proper map always exists and by Theorem 5.10 of [5] any map can be uniformly approximated by an immersion provided some immersion exists. Since the uniform approximation of a proper map is proper, the conclusion follows (cf. the proof of Lemma 5 in \$3).

The remainder of this paper is organized as follows: In \$1, the concept of an end of a manifold with volume form having finite or infinite volume is discussed and an example given to show the role of the end condition in 
Theorem 1. In $\$ 2$, the proof of Theorem 1 is discussed briefly and in $\$ 3$ the proof of Theorem 2 is discussed.

In $\$ 4$, generalizations of Theorems 1 and 2 to the case of noncompact manifolds with boundary and to the case of odd forms on nonorientable manifolds are discussed.

Throughout the paper a manifold is taken to be connected by definition, unless otherwise specified.

Theorems 1 and 2 were announced in [3]. The authors are indebted to the Mathematics Institute of the University of Copenhagen for hospitality during the period when their work on this paper was initiated. The authors are also indebted to M. Hirsch for helpful discussions on immersion theory and to the referee for some useful suggestions.

1. Ends of manifolds and the action of the diffeomorphism group on volume forms. Suppose $M$ is a noncompact paracompact manifold. If $K$ and $K^{\prime}$ are compact subsets of $M$ with $K \subset K^{\prime}$, then any component $C^{\prime}$ of $M-K^{\prime}$ is contained in a unique component $C$ of $M-K$. An end of $M$ is an element of the inverse limit system $\{K$, components of $M-K\}$ indexed by $\{K \mid K$ compact subset of $M$ \} directed by inclusion as indicated.

Suppose $M$ is orientable and has a volume form $\omega$. Then an end $\varepsilon$ of $M$ has by direction finite volume if there is a compact set $K$ such that the volume of the component of $M-K$ containing the end $\varepsilon$ is finite. An end has by definition infinite volume if it does not have finite volume. Note that an end with finite volume does not have a numerical (finite) volume: The volume of the component of $M-K$ containing a finite-volume end depends on the choice of $K$; in fact, by a suitable choice of $K$, this volume can be made arbitrarily small.

To see the role of the end condition in Theorem 1, consider the case

$$
M=R^{2}-\{(0,0)\}, \quad \omega=d x \wedge d y, \quad \tau=(d x \wedge d y) / F\left(x^{2}+y^{2}\right),
$$

where $F: R^{+} \rightarrow R^{+}$is a $C^{\infty}$ function such that

$$
F(r)=1 \text { if } r>1 \text { and } F(r)=r^{2} \text { if } 0<r<\frac{1}{2} .
$$

Clearly $\int_{M} \omega=\int_{M} \tau=+\infty$. However, the end condition is violated: $R^{2}-$ $\{(0,0)\}$ has two ends, one identifiable with $(0,0)$, the other with the "point at infinity" in $R^{2}$. The "point at infinity" end has infinite $\tau$ volume and infinite $\omega$ volume as well. However, the $(0,0)$-end has finite $\omega$ volume but infinite $\tau$ volume.

It is in fact the case that there is no diffeomorphism $\varphi: M \rightarrow M$ such that $\varphi^{*} \tau=\omega$. To see this observe that $\tau$ has the property that any embedded circle $\left(S^{1}\right)$ in $M$ which is not homologous to 0 divides $M$ into two components, each having infinite $\tau$-volume. Clearly this property is diffeomorphism invariant, 
i.e., the same property will hold for $\varphi^{*} \tau$ for any diffeomorphism $\varphi: M \rightarrow M$. But $\omega$ does not have this property: one component of $M-\left\{(x, y) \mid x^{2}+y^{2}=\right.$ 1) has finite $\omega$-volume. Thus there is no diffeomorphism $\varphi: M \rightarrow M$ such that $\varphi^{*} \tau=\omega$.

It can happen that a diffeomorphism $\varphi: M \rightarrow M$ can exist with $\varphi^{*} \omega=\tau$ even if $\omega$ and $\tau$ do not satisfy the end condition. What is required is that there should be a diffeomorphism acting on the ends so as to make the end condition satisfied. Precisely, there is a diffeomorphism $\varphi: M \rightarrow M$ (for any noncompact paracompact $M$ ) such that $\varphi^{*} \omega=\tau$ if and only if $\int_{M} \omega=\int_{M} \tau$ $<+\infty$ and there is a diffeomorphism $\varphi_{1}: M \rightarrow M$ such that $\varphi_{1}^{*} \omega$ and $\tau$ satisfy the end condition of Theorem 1, i.e., each end if $M$ has its $\varphi_{1}^{*} \omega$ and $\tau$ volumes either both finite or both infinite. An example of this possibility (where $\varphi_{1}$ cannot be the identity) is obtained by again taking $M=R^{2}-$ $\{(0,0)\}$ and taking

$$
\omega=(d x \wedge d y) /\left(x^{2}+y^{2}\right)^{2} \text { and } \tau=(d x \wedge d y) .
$$

Here $\omega$ and $\tau$ have each one infinite and one finite volume but the finiteness occurs for different ends in the two cases. But if $\varphi_{1}: M \rightarrow M$ is the diffeomorphism $\varphi_{1}((x, y))=(x, y) /\left(x^{2}+y^{2}\right)$ then $\varphi_{1}^{*} \omega$ and $\tau$ both have the $(0,0)$ end finite volume and the "point at infinity" end infinite volume. This follows from observing that $\varphi_{1}$ interchanges the two ends. It is not always the case (for general $M$ ) that the diffeomorphism group acts transitively on the set of ends. Thus the existence of a suitable $\varphi_{1}$ is usually a more subtle question than the question of whether the cardinality of the set of finite-volume ends for $\omega$ equals the cardinality of the set of finite-volume ends for $\tau$ and similarly for infinite-volume ends.

2. The proof of Theorem 1. The following lemmas will be used in the proofs of both Theorem 1 and Theorem 2 .

LEMMA 1. If $\omega$ and $\tau$ are two volume forms on an oriented manifold $M$ and if there is a connected compact set $K$ with the properties that the support of $\omega-\tau$ is contained in the interior $\dot{K}$ of $K$ and that $\int_{K} \omega=\int_{K} \tau$, then there is $a$ diffeomorphism $\varphi: M \rightarrow M$ such that $\varphi \mid(M-K)$ is the identity mapping and such that $\varphi^{*} \omega=\tau$.

In Lemma 1, the support of $\omega-\tau$ is as usual the closure of the set $\{p \in M \mid(\omega-\tau)(p) \neq 0\}$. Lemma 1 is an immediate consequence of the argument used by Moser [6] to establish the theorem for compact manifolds referred to in the introduction of this paper.

LEMMA 2. Suppose that $M$ is an oriented manifold and that $\omega$ and $\tau$ are volume forms on $M$. Suppose also that $N$ is a connected codimension 1 
submanifold of $M$ and $U$ is a tubular neighborhood of $N$ (i.e. a neighborhood which is diffeomorphic to $N \times(-1,1)$ under a diffeomorphism which takes $N$ to $N \times 0)$. Let $U_{+}$and $U_{-}$be the components of $U-N$. Then there is a diffeomorphism $\varphi: M \rightarrow M$ such that $\varphi \mid(M-U)$ is the identity, $\varphi^{*} \omega=\tau$ on some neighborhood $V \subset U$ of $N$, and $\int_{U_{+}} \varphi^{*} \omega=\int_{U_{+}} \omega$ and $\int_{U_{-}} \varphi^{*} \omega=\int_{U_{-}} \omega$.

Proof of Lemma 2. Let $W$ be a tubular neighborhood of $N$ having compact closure $\bar{W} \subset U$. Let $F, G: M \rightarrow R$ be $C^{\infty}$ functions with support in $W$ and with the properties that $F=1, G=1$ in a neighborhood of $N, G<1$ on $M$, and

$$
\int_{U_{+} \cap W}(1-G) \omega+F \tau=\int_{U_{+} \cap W} \omega
$$

and

$$
\int_{U_{-} \cap W}(1-G) \omega+F_{\tau}=\int_{U_{-} \cap W} \omega .
$$

Since the support of $\omega-((1-G) \omega+F \tau)$ is contained in $W$ and hence in the interior of the compact set $\bar{W}$ it follows from Lemma 1 that there is a diffeomorphism $\varphi: M \rightarrow M$ with $\varphi \mid(M-\bar{W})=$ identity and $\varphi^{*} \omega=(1-$ $G) \omega+F \tau$.

LEMMA 3. Suppose $M$ is a noncompact orientable manifold of dimension $n$ and $\left\{K_{i} \mid i=1,2, \ldots\right\}$ is a sequence of $n$-dimensional compact connected submanifolds-with-boundary such that $\cup_{i=1}^{+\infty} K_{i}=M$ and $K_{i} \cap K_{j}$ for all $i, j, i \neq j$, is either empty or is an $(n-1)$-dimensional submanifold of $M$ which is contained in the boundary of $K_{i}$ and also in the boundary of $K_{j}$. Suppose also that $\omega$ and $\tau$ are volume forms on $M$ such that $\int_{K_{i}} \omega=\int_{K_{i}} \tau$ for each $i=1,2, \cdots$. Then there is a diffeomorphism $\varphi: M \rightarrow M$ such that $\varphi^{*} \omega=\tau$.

Proof of Lemma 3. According to Lemma 2 there is a diffeomorphism $\psi$ : $M \rightarrow M$ which equals the identity outside the union of disjoint tubular neighborhoods of the connected components of the boundaries of the $K_{i}$ with the properties that $\psi^{*} \omega=\tau$ near the union of the boundary components and $\int_{K_{1}} \psi^{*} \omega=\int_{K_{1}} \tau$ for all $i=1,2, \ldots$ (The last condition here follows from the final two equalities in the conclusion of Lemma 2: the total volume on either side of $N$ is unchanged by the diffeomorphism $\varphi$ there.) By Lemma 1 there is, for each $i=1,2, \ldots$, a diffeomorphism $\varphi_{1}: K_{i} \rightarrow K_{i}$ such that $\varphi_{i}=$ identity in a neighborhood of the boundary of $K_{i}$ and $\varphi_{i}^{*}\left(\psi^{*} \omega\right)=\tau$ on $K_{i}$. Clearly there is a diffeomorphism $\varphi_{\infty}: M \rightarrow M$ such that $\varphi_{\infty} \mid K_{i}=\varphi_{i}$. Then $\left(\psi \varphi_{\infty}\right)^{*} \omega$ $=\tau$.

For the proof of Theorem 1, one more lemma is needed: 
LEMMA 4. Let $K$ be a compact subset of a noncompact oriented manifold $M$ with volume form $\omega$. Then a connected component $C$ of $M-K$ has infinite volume if and only if $C$ contains an end of $K$ that has infinite volume.

Proof of Lemma 4. Clearly if $C$ contains an end of infinite volume then $C$ has infinite volume. To prove the converse, suppose $C$ has infinite volume. Let $\left\{K_{i} \mid i=1,2, \ldots\right\}$ be a sequence of compact subsets of $M$ having the properties that $K_{1}=K, \cup K_{i}=M, K_{i} \subset \dot{K}_{i+1}$ for all $i=1,2, \ldots$ and for all $i=2,3, \ldots, M-K_{i}$ has only finitely many components all of which have noncompact closures. (Such a sequence is easily constructed.) Now consider the components of $M-K_{2}$ which lie in $C$. Clearly one of these components has infinite volume for otherwise the facts that there are only finitely many of these components and that $K_{2} \cap C$ has compact closure would imply that $C$ has finite volume. Let $C_{2}$ be one of the components of $M-K_{2}$ which has infinite volume. Repeating the argument one obtains a component $C_{3}$ of $M-K_{3}$ which is contained in $C_{2}$ and which has infinite volume, etc. The sequence $C \supset C_{2} \supset C_{3} \supset C_{4} \supset \cdots$ of components of $M-K, M-K_{2}$, $M-K_{3}, M-K_{4}, \ldots$ gives an end with infinite volume.

Proof of Theorem 1. Choose a $C^{\infty}$ function $F: M \rightarrow R$ such that for all $\alpha \in R, F^{-1}((-\infty, \alpha])$ is compact and such that each positive integer is a noncritical value of $F$. Define a sequence of compact subsets of $M$ as follows: Let $p$ be a point of $F^{-1}((-\infty,+1])$, which is assumed without loss of generality to be nonempty. Put $M_{1}=$ the union of the connected component $C$ of $F^{-1}((-\infty,+1])$ which contains $p$ with the components of $M-C$ which have compact closure. Put $M_{2}=$ the connected component of $F^{-1}((-\infty,+2])$ which contains $M_{1}$ union the closure-compact components of the complement of that component, etc. Clearly, for each $i=1,2, \ldots, M_{i}$ is connected; also for each $i=1,2, \ldots, M_{i} \subset \dot{M}_{i+1}$; and $\cup_{i=1}^{+\infty} M_{i}=M$. Furthermore, for each $i=1,2, \ldots$, every component of $M-M_{i}$ has noncompact closure.

The proof will consist of an iterated construction of volume forms $\omega_{1}$ and $\tau_{1}, \omega_{2}$ and $\tau_{2}$, etc. and diffeomorphisms $\varphi_{1}: M \rightarrow M$ and $\psi_{1}: M \rightarrow M, \varphi_{2}$ and $\psi_{2}$, etc. such that $\varphi_{1}^{*} \omega=\omega_{1}$ and $\psi_{1}^{*} \tau=\tau, \varphi_{2}^{*} \omega_{1}=\omega_{2}$ and $\psi_{2}^{*} \tau_{1}=\tau_{2}$, etc. This iterative construction is as follows:

Set $i_{1}=1$. Choose $\omega_{1}$ and $\tau_{1}$ volume forms on $M$ with the following properties:

(a) the supports of $\omega-\omega_{1}$ and $\tau-\tau_{1}$ are compact;

(b) $\int_{M_{i_{1}}} \omega_{1}=\int_{M_{i_{1}}} \tau_{1}=\max \left(\int_{M_{i_{1}}} \omega, \int_{M_{i_{1}}} \tau\right)$;

(c) $\int_{\operatorname{supp}\left(\omega-\omega_{1}\right)} \omega_{1}=\int_{\operatorname{supp}\left(\omega-\omega_{1}\right)} \omega$ and $\int_{\operatorname{supp}\left(\tau-\tau_{1}\right)} \tau_{1}=\int_{\operatorname{supp}\left(\tau-\tau_{1}\right)} \tau$

where $\operatorname{supp}\left(\omega-\omega_{1}\right)=\operatorname{support}$ of $\left(\omega-\omega_{1}\right)$ and $\operatorname{supp}\left(\tau-\tau_{1}\right)=$ support of $\left(\tau-\tau_{1}\right)$

(d) $\int_{C} \omega_{1}=\int_{C} \tau_{1}$ for each component $C$ of $M-M_{i_{1}}$; 
(e) for each component $C$ of $M-M_{i_{1}}, C-\left(\operatorname{supp}\left(\omega-\omega_{1}\right) \cup \operatorname{supp}\left(\tau-\tau_{1}\right)\right)$ is connected.

That such choices are possible is clear since the only condition involving nonfinite volumes is (d), and the possibility of that condition being satisfied is guaranteed by the end condition hypothesis combined with Lemma 4.

Informally, the $\omega_{1}$ and $\tau_{1}$ are $\omega$ and $\tau$ with some volume moved around so that $\omega_{1}$ and $\tau_{1}$ have equal total on $M_{i_{1}}=M_{1}$ and so that the components of $M-M_{i_{1}}$ have equal totals. Note that if the end condition hypothesis were omitted then this equal totals on the components of $M-M_{i_{1}}$ might not be arrangeable consistently with condition (a).

Now there exist by Lemma 1 diffeomorphisms $\varphi_{1}: M \rightarrow M$ and $\psi_{1}: M \rightarrow M$ such that $\varphi_{1}^{*} \omega=\omega_{1}$ and $\psi_{1}^{*} \tau=\tau_{1}$ and such that $\varphi_{1}$ and $\psi_{1}=$ identity outside arbitrarily small neighborhoods $U_{1}$ and $V_{1}$ of $\operatorname{supp}\left(\omega-\omega_{1}\right)$ and $\operatorname{supp}\left(\tau-\tau_{1}\right)$. In particular, these neighborhoods $U_{1}$ and $V_{1}$ can be taken so that they have compact closure and so that the union of their closures does not disconnect any component of $M-M_{i}$.

For the second stage of the construction, pick a positive integer $i_{2}>i_{1}+2$ and so large that $\varphi_{1}$ and $\psi_{1}=$ the identity on $M_{i_{2}-2}$. Choose volume forms $\omega_{2}$ and $\tau_{2}$ such that:

(a) $\operatorname{supp}\left(\omega_{1}-\omega_{2}\right)$ and $\operatorname{supp}\left(\tau_{1}-\tau_{2}\right)$ are compact; $\operatorname{supp}\left(\omega_{1}-\omega_{2}\right) \cap M_{i_{1}}$ is empty; and $\operatorname{supp}\left(\tau_{1}-\tau_{2}\right) \cap M_{i_{1}}$ is empty; and $\operatorname{supp}\left(\omega_{1}-\omega_{2}\right) \cap \bar{U}_{1}$ is empty and $\operatorname{supp}\left(\tau_{1}-\tau_{2}\right) \cap \bar{V}_{1}$ is empty;

(b) $\int_{C} \omega_{2}=\int_{C} \tau_{2}=\max \left(\int_{C} \omega_{1}, \int_{C} \tau_{1}\right)$ for each component $C$ of $M_{i_{2}}-M_{i_{1}}$;

(c) $\int \omega_{1}=\int \omega_{2}$ over $\operatorname{supp}\left(\omega_{1}-\omega_{2}\right), \int \tau_{1}=\int \tau_{2}$ over $\operatorname{supp}\left(\tau_{1}-\tau_{2}\right)$;

(d) $\int_{C} \omega_{2}=\int_{C} \tau_{2}$ for each component $C$ of $M-M_{i_{2}}$;

(e) for each component $C$ of $M-M_{i_{2}}, C-\left(\operatorname{supp}\left(\omega_{1}-\omega_{2}\right) \cup \operatorname{supp}\left(\tau_{1}-\right.\right.$ $\left.\tau_{2}\right)$ ) is connected.

Again one then obtains diffeomorphisms $\varphi_{2}: M \rightarrow M, \psi_{2}: M \rightarrow M$ such that $\varphi_{2} \omega_{1}=\omega_{2}$ and $\psi_{2} \tau_{1}=\tau_{2}$. These diffeomorphisms can and are to be taken to be equal to the identity outside neighborhoods $U_{2}$ and $V_{2}$ of $\operatorname{supp}\left(\omega_{1}-\omega_{2}\right)$ and $\operatorname{supp}\left(\tau_{1}-\tau_{2}\right)$, respectively, which are so small that their closures are disjoint respectively from $\bar{U}_{1}$ and $\bar{V}_{1}$ and so that $M_{i_{1}} \cap U_{2}$ is empty and $M_{i_{1}} \cap V_{2}$ is empty. Also, the neighborhoods $U_{2}$ and $V_{2}$ are to be chosen so that for each component $C$ of $M-M_{i_{2}}, C$ is not disconnected by the union of $\bar{U}_{2}$ and $\bar{V}_{2}$.

The construction now continues inductively. The $\varphi_{i}$ and $\psi_{i}$ were chosen so that there are diffeomorphisms $\varphi_{\infty}: M \rightarrow M$ and $\psi_{\infty}: M \rightarrow M$ such that $\varphi_{\infty} \mid U_{i}=\varphi_{i}$ and $\psi_{\infty} \mid V_{i}=\psi_{i}$ for all $i$ and $\varphi_{\infty} \mid\left(M-\cup_{i=1}^{\infty} U_{i}\right)=$ identity and $\psi_{\infty} \mid\left(M-\cup_{i=1}^{\infty} V_{i}\right)=$ identity. Now the volume forms $\varphi_{\infty}^{*} \omega$ and $\psi_{\infty}^{*} \tau$ have equal total volumes on $M_{i_{1}}$ and on each component of $M_{i_{k}}-M_{i_{k-1}}, k=$ $2,3,4, \ldots$ Lemma 3 implies that there is a diffeomorphism $\eta: M \rightarrow M$ such 
that $\eta^{*}\left(\psi_{\infty}^{*} \tau\right)=\varphi_{\infty}^{*} \omega$. Thus $\tau=\left(\varphi \eta_{\infty}^{-1} \psi_{\infty}^{-1}\right)^{*} \omega$.

3. The proof of Theorem 2. The proof of Theorem 2 will be given in this section. Note that Theorem 2 does not follow from Theorem 1 as the embedding (immersion) result for compact manifolds follows from Moser's theorem: the end condition in Theorem 1 and also the fact that an infinite volume embedding or immersion cannot be converted to a finite one by multiplication by a constant necessitate a separate argument.

The proof of Theorem 2 depends upon a preliminary result about proper embeddings and immersions in euclidean space.

LEMMA 5. If $M$ is a noncompact oriented manifold of dimension $>1$, if there is a proper embedding (immersion) of $M$ in a euclidean space $R^{k}, k>\operatorname{dim} M$ +1 , and if $\omega$ is a volume form on $M$ then there is a proper embedding (immersion) $E: M \rightarrow R^{k}$ such that $\omega_{E}<\omega$, where $\omega_{E}$ is the volume form of the Riemannian metric induced by $E$.

Proof of Lemma 5. First, suppose that there is a proper embedding of $M$ in $R^{k}$; it will be shown that there is a proper embedding $E_{1}: M \rightarrow R^{k}$ such that $E_{1}(M)$ is contained in an open half-space of $R^{k}$. For this purpose, the cases $k>3$ and $k=3$ need to be considered separately:

If $k>3$, then any proper semi-infinite embedded curve $C:[0,+\infty) \rightarrow R^{k}$ is unknotted, so for instance there is a diffeomorphism $\varphi: R^{k} \rightarrow R^{k}$ such that $\varphi \circ C:[0,+\infty) \rightarrow R^{k}$ is the map $(\varphi \circ C)(t)=(t, 0, \ldots, 0)$. Since it is easy to see that there is a proper semi-infinite embedded curve in $R^{k}-E(M)$, it follows that there is a proper embedding $E_{0}: M \rightarrow R^{k}$ such that $E_{0}(M) \cap$ $\{(t, 0, \ldots, 0) \mid t>0\}$ is empty, namely $\varphi \circ E$. Since $E_{0}$ is proper, there is a tube-shaped neighborhood $U$ of $\{(t, 0, \ldots, 0) \mid t>0\}$ such that $\bar{U} \cap E_{0}(M)$ is empty. But there is a diffeomorphism $\varphi_{1}: R^{k} \rightarrow R^{k}$ such that $\varphi_{1}(U)$ is an open half-space. Then $\varphi_{1} \circ E_{0}$ is a proper embedding with $\left(\varphi_{1} \circ E_{0}\right)(M)$ contained in the open half-space $R^{k}-\varphi_{1}(\bar{U})$. If $k=3$, then the dimension of $M=2$. Let $F: M \rightarrow R$ be a function with the properties that $F^{-1}((-\infty, \alpha])$ is compact for all $\alpha \in R$, each $i=1,2, \ldots$ is a noncritical value of $F$, and $F(p)>1 / 2$ for all $p \in M$. Each of the sets $M_{i}=F^{-1}([i-$ $1, i]), i=1,2, \ldots$, is a compact 2-dimensional oriented manifold with boundary, i.e. a finite union of spheres with a certain number of handles attached and a certain number of tubes-with-boundary attached. It is easy to see that each $M_{i}$ can be embedded between $\{(x, y, i-1) \mid x, y \in R\}$ and $\{(x, y, i) \mid x, y \in R\}$ in such a way that the boundary components of $M_{i}$ on which $F=i-1$ are embedded in $\{(x, y, i-1)\}$ and those on which $F=i$ are embedded in $\{(x, y, i)\}$. Clearly, also, these embeddings can be chosen so that they fit together to form an embedding of $M$ itself which lies in the half-space $\{(x, y, z) \mid z>0\}$. 
Let now $E_{1}: M \rightarrow R^{k}$ be a proper embedding such that $E_{1}(M)$ is contained in a half-space $H$. Choose a diffeomorphism $\varphi_{2}: R^{k} \rightarrow R^{k}$ which maps $H$ into a subset $\left\{\left(x_{1}, x_{2}, \ldots, x_{n}\right) \mid x_{n}>0, \sum_{i=1}^{n-1} x_{i}^{2}<1\right\}$; then $\varphi_{2} \circ E_{1}: M \rightarrow R^{k}$ is a proper embedding with

$$
\left(\varphi_{2} \circ E_{1}\right)(M) \subset\left\{\left(x_{1}, x_{2}, \ldots, x_{n}\right) \mid x_{n}>0, \sum_{i=1}^{n-1} x_{i}^{2}<1\right\} .
$$

Now define a diffeomorphism $\varphi_{3}: R^{k} \rightarrow R^{k}$ by

$$
\left(x_{1}, \ldots, x_{n-1}, x_{n}\right) \rightarrow\left(F\left(x_{n}\right) x_{1}, \ldots, F\left(x_{n}\right) x_{n-1}, x_{n}\right)
$$

where $F$ is a positive $C^{\infty}$ function with $F\left(x_{n}\right)<1$ for all $x_{n}$. Note that, because the dimension of $M$ is at least 2 , if $\tau^{\prime}=$ the volume form induced by $\varphi_{2} \circ E_{1}$ and $\tau^{\prime \prime}=$ the volume form induced by $\varphi_{3} \circ \varphi_{2} \circ E_{1}$ then

$$
\tau^{\prime \prime}(p)<F\left(z\left(\left(\varphi_{2} \circ E_{1}\right)(p)\right)\right) \tau^{\prime}(p) .
$$

Since $\varphi_{2} \circ E_{1}$ is proper, it follows that $F$ can be chosen so that $\tau^{\prime \prime}<\omega$.

The arguments just given for the case of embeddings hold mutatis mutandis for the case of immersions with one exception: if $E: M^{n} \rightarrow \mathrm{R}^{n+1}$ is a proper immersion then $\mathrm{R}^{n+1}-F(M)$ may fail to contain a proper image of $[0,+\infty)$. To avoid this difficulty, one can construct the required proper immersion image in a half-space by choosing a transversal hyperplane, reflecting the part of the original immersion that goes into a fixed half-space across the hyperplane and smoothing along the resulting "fold".

Alternatively, one can treat the immersion case by applying the general theory of immersions of Hirsch [5] as follows: Let $F: M \rightarrow \mathbf{R}$ be a $C^{\infty}$ function with $F^{-1}((-\infty, \alpha])$ compact for all $\alpha \in \mathbf{R}$ (as before). The map $F_{1}$ : $M \rightarrow \mathbf{R}^{N}$ defined by

$$
F_{1}(p)=(0, \ldots, 0, F(p)), \quad p \in M,
$$

is proper. By Theorem 5.10 of [5], the existence of any immersion (proper or not, in fact) $E: M \rightarrow \mathbf{R}^{N}$ implies (since $N>n$ ) that there is an immersion $E_{1}$ : $M \rightarrow \mathbf{R}^{N}$ which approximates $F_{1}$, say $\left\|F_{1}(p)-E_{1}(p)\right\|<1$ for all $p \in M$. Then $E_{1}$ is proper and $E_{1}(M)$ is contained in the half-space $\left\{\left(x_{1}, \ldots, x_{N}\right) \mid x_{N}\right.$ $\left.>1+\min _{M} F\right\}$. The rest of the proof is as in the embedding case.

Proof of Theorem 2. Let $E_{0}: M \rightarrow R^{k}$ be a proper embedding (immersion) of $M$ in $R^{k}$ such that $\omega_{E_{0}}<\omega$ : the existence of $E_{0}$ is guaranteed by Lemma 5. Choose a function $F: M \rightarrow R$ as in previous proofs (i.e. $F$ with $F>1 / 2, i=1,2, \ldots$, noncritical values, and with $F^{-1}((-\infty, \alpha])$ compact for all $\alpha \in R)$. Then define a sequence $M_{i}, i=1,2, \ldots$, by $M_{i}=F^{-1}([i-$ $1, i]$ ), each $M_{i}$ being a compact submanifold with boundary. Let $M_{j}^{\prime}, j=$ $1,2, \ldots$, be the compact connected submanifolds with boundary which are 
the components of the $M_{i}$ 's. According to Lemma 3, the theorem will follow if there exists an embedding $E_{1}: M \rightarrow R^{k}$ such that for each $j, \int_{M_{j}^{\prime}} \omega_{E_{1}}=\int_{M_{j}^{\prime}} \omega$ : For then there is a diffeomorphism $\varphi: M \rightarrow M$ such that $\varphi^{*} \omega_{E_{1}}=\omega$ and thus $\omega_{E_{1} \circ \varphi}=\omega$ and $E_{1} \circ \varphi$ is the required volume-preserving embedding (immersion). Now $\int_{M^{\prime}} \omega_{E_{0}}<\int_{M^{\prime}} \omega$ so what is needed is a perturbation of $E_{0}$ which increases the induced volume. Such perturbations which preserve the property of being a proper embedding (immersion) can be constructed using a technique developed in [7].

Specifically, the construction is as follows: The global perturbation is obtained from a sequence of local ones. For each $j=1,2, \ldots$, let $p_{j}$ be a point of $\dot{M}_{j}^{\prime}$; a perturbation of the embedding (immersion) in a neighborhood of each $p_{j}$ will be found so that the required conditions are satisfied. For this purpose, for each $j=1,2, \ldots$, let $N_{j}$ be a unit vector normal at $p_{j}$ to the image of $M$. Choose a neighborhood $U_{j}$ of $p_{j}$ in $M$ such that $\bar{U}_{j} \subset \dot{M}_{j}^{\prime}$ and such that the composition of $E_{0}$ with the orthogonal projection on the orthogonal complement of $N_{j}$ is an embedding of $\bar{U}_{j}$. Then for any $C^{\infty}$ function $F_{j}: M \rightarrow R$ with supp $F_{j} \subset U_{j}$, and $\left|F_{j}\right|<1$, the mapping $E_{0}+F_{j} N_{j}$ defined by

$$
\left(E_{0}+\sum_{j} F_{j} N_{j}\right)(p)=E_{0}(p)+\sum_{j} F_{j}(p) N_{j}
$$

is a proper immersion (note that the vector sum is finite, having in fact at most two nonzero terms). Furthermore, if $E_{0}$ is an embedding, there is a sequence $\varepsilon_{1}, \varepsilon_{2}, \varepsilon_{3}, \ldots$ of positive numbers such that if $\left|F_{j}\right|<\varepsilon_{j}$ then $E_{0}+$ $\Sigma_{j} F_{j} N_{j}$ is an embedding. On the other hand, by choosing the $F_{j}$ 's to oscillate rapidly while still satisfying $\left|F_{j}\right|<1$ (and $\left|F_{j}\right|<\varepsilon_{j}$ in the embedding case) it can clearly be arranged that

$$
\int_{M_{j}^{\prime}} \omega_{E_{0}+\Sigma, F_{j} N}>\int_{M_{j}^{\prime}} \omega
$$

Consider $\omega_{E_{0}+\Sigma, \lambda F_{j}, N}$ where $0<\lambda_{j}<1$. Clearly the integral of this volume form over $M_{j}^{\prime}$ is a continuous function of $\lambda_{j}$ (and is independent of $\lambda_{k}, k \neq j$ ). On the other hand, if $\lambda_{j}=1$ then the integral over $M_{j}^{\prime}$ is $>\int_{M_{j}^{\prime}} \omega$ while if $\lambda_{j}=0$, the integral is $<\int_{M_{j}} \omega$. Hence there exist $\lambda_{j}, j=1,2, \ldots, 0<\lambda_{j}<1$, such that for all $j=1,2, \ldots$

$$
\int_{M_{j}^{\prime}} \omega_{E_{0}+\Sigma \lambda_{j} F_{j} N_{j}}=\int_{M_{j}^{\prime}} \omega
$$

as required. 


\section{Generalizations to noncompact manifolds with boundary and nonorientable} manifolds. The results of the previous sections have generalizations to manifolds with boundary and to odd forms on nonorientable manifolds (possibly with boundary). These generalizations and their proof are formally virtually identical to the theorems as given, and the detailed formulations and proof of these results will be left to the reader. A few comments are in order, however:

In the (orientable) manifold with boundary case, in attempting to transform one volume form $\omega$ to another $\tau$ via a diffeomorphism, one can first find a diffeomorphism $\varphi_{1}: M \rightarrow M$ fixing the boundary $\partial M$ of $M$ pointwise and equal to the identity except near $\partial M$ such that $\varphi_{1}^{*} \omega=\tau$ near $\partial M$. Then proceeding as before (provided the appropriate total volume and end conditions are satisfied) one need only consider diffeomorphisms with support away from $\partial M$, and one finds $\varphi: M \rightarrow M$ such that $\varphi^{*} \omega=\tau$ and $\varphi \mid \partial M=$ identity. To obtain $\varphi_{1}$ choose a tubular neighborhood $U \simeq \partial M \times[0,1)$ of $M$ and let $\varphi_{1}((p, t))=(p, f(p, t))$ where $f(p, 0)=0$ and $\partial f / \partial t=\tau(p, t) / \omega(p, t)$. This gives $\varphi_{1}$ near $\partial M$ and $\varphi_{1}$ is extended globally by an obvious procedure.

To turn to the nonorientable case, recall that if $M$ is nonorientable then a nowhere vanishing odd form $\omega$ on $M$ is by definition a volume form $\tilde{\omega}$ on $\tilde{M}$, the orientable double cover of $M$, such that $I^{*} \tilde{\omega}=-\tilde{\omega}$, where $I$ is the nontrivial covering transformation (with $I^{2}$ identity) on $\tilde{M}$. To prove the desired generalizations to this case, one can proceed as follows: Choose a connected smoothly bounded open set $D$ in $\tilde{M}$ such that $D \cap I(D)=\partial D$ and $D \cup I(D)=M$. Then carry out the constructions of the previous sections on $D$ and extend to $I(D)$ by applying $I$. The result clearly has the required $I$-equivariance. The only delicate point is to be sure of smoothness across $\partial D$. For this point, one must choose an equivariant tubular neighborhood of $\partial D$ and use this to make the constructions operating near $\partial D$ $I$-equivariant. The existence of a fundamental domain $D$ as described follows, as kindly pointed out to the authors by W. Meeks and B. O'Neill, from considering the classifying map of the $\mathrm{Z}_{2}$ orientation bundle $\tilde{M} \rightarrow M$, say $G$ : $M \rightarrow \mathbf{R} P^{N}, N$ large. If $G$ is taken transversal to a hyperplane $P$ in $\mathbf{R} P^{N}$, then $G^{-1}(P)$ is a smooth hypersurface and $M-G^{-1}(P)$ is orientable. There is an open subset $D$ of $M$ which is maximal among those connected open subsets of $M$ which are orientable and the boundary of which is a union of components of $G^{-1}(P)$. Such a $D$ has the required properties.

\section{REFERENCES}

1. D. V. Anosov and A. B. Katok, New examples in smooth engodic theory. Engodic diffeomorphisms, Trans. Moscow Math. Soc. 23 (1970), 1-35.

2. A. Banyaga, Formes-volume sur les variétés à bord, Enseignement Math. (2) 20 (1974), 127-131.

3. R. E. Greene and K. Shiohama, Volume-preserving diffeomorphisms and embeddings of noncompact manifolds. Notices Amer. Math. Soc. 23 (1976), Abstract A-446. 
4. M. L. Gromov, Smoothing and inversion of differential operators, Math. USSR-Sb. 17 (1972), $381-435$.

5. M. Hirsch, Immersions of manifolds, Trans. Amer. Math. Soc. 93 (1959), 242-276.

6. J. Moser, On the volume elements on a manifold, Trans. Amer. Math. Soc. 120 (1965), 286-294.

7. J. Nash, $C^{1}$ isometric embeddings, Ann. of Math. (2) 60 (1954), 383-396.

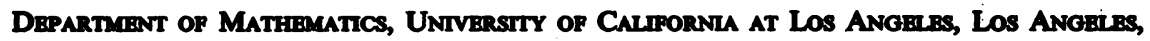
Calmornin 90024

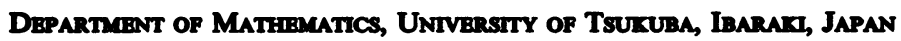

